\title{
On the study of cyclic plasticity behaviour of primary electrode particle for lithium-ion battery
}

\author{
Xuanchen Zhu', Haofeng Chen ${ }^{1,2, *}$, Weiling Luan ${ }^{2, *}$ \\ ${ }^{1}$ Department of Mechanical \& Aerospace Engineering, University of Strathclyde, G1 1XJ, UK \\ ${ }^{2}$ Key Laboratory of Pressure Systems and Safety (MOE), School of Mechanical and Power Engineering, East
}

China University of Science and Technology, 200237, China

\begin{abstract}
The mechanical failure of battery electrode, caused by the cyclic diffusion-induced stress, is generally thought to be a direct reason leading to the loss of capacity and deterioration of performance for Liion battery. In the present work, the cyclic plasticity behaviour of primary electrode particle under electrochemical-mechanical condition is evaluated by using the Linear Matching Method (LMM). The coupled diffusion-stress analysis of electrode particle during lithiation-delithiation process is performed with the developed finite element subroutines as the groundwork for the subsequent cyclic plasticity assessment. The shakedown boundaries are established for electrode particle considering the variation of particle diameter by means of the LMM. ABAQUS full cyclic incremental analysis is employed to verify the applicability and accuracy of the obtained boundary. Two types of failure mechanisms known as low-cycle fatigue and ratcheting are investigated for particle subjected to loading history out of shakedown boundary. Steady state cycle analysis is conducted to study the generation of plastic strain range of particle under certain electrochemical-mechanical condition and the corresponding low-cycle fatigue damage is assessed. Different forms of ratcheting boundaries for electrode particle with various dimensions are created and the influences of cyclic current density and mechanical load on cyclic plasticity limits are clarified. The established critical failure diagrams will offer the instructional information regarding the design of electrode material in microscale within designated electrochemical condition.
\end{abstract}

Keywords: Li-ion battery electrode, Diffusion induced stress, Cyclic plasticity behaviour, The linear matching method

\section{Introduction}

Lithium-ion battery is widely regarded as a promising solution for coping with energy crisis and has been commonly used for portable electronics, electric vehicles, aerospace applications, etc. [1, 2, 3]. Working as a cyclic energy system, the battery runs on the basis of Li-ion inserting into and extracting from electrode. The swell and shrink of electrode caused by this lithiation-delithiation behaviour will result in the diffusion-induced stress (DIS) $[4,5,6]$, which has been investigated by a large number of researchers with adopting various methods. Christensen and Newman [7] have derived a mathematical model for studying the concentration and stress fields of particle structure under lithiation-delithiation condition. Zhang et al. have studied the DIS generated in an ellipsoidal particle by using the finite difference method and finite element methods [8]. Weng et al. [9] have presented an analytical model for studying the diffusion-induced stress in nanowire electrode under galvanostatic and potentiostatic operations. Cui et al. have established a stress-related chemical potential and applied it to investigate the DIS within silicon particle [10]. Korsunsky and co-workers

\footnotetext{
* Corresponding author.

Email: haofeng.chen@strath.ac.uk (H Chen), luan@ecust.edu.cn (W Luan) Tel: +44(0) 1415482036
} 
[11] presented an explicit closed form approximate solution for intercalation stress in spherical particle, which provides a basis for the subsequent damage assessment of Li-ion battery electrode. In our preliminary work, we [12] have developed the diffusion driven approach and the chemical potential driven approach in commercial finite element software ABAQUS to study the evolution of diffusion-induced stress within primary electrode particle. Besides, for some materials, the volumetric change caused by lithiation-delithiation behaviour is relatively large and large deformation theory is required to be applied. When studying the mechanical failure in silicon electrode, An et al. presented a finite element based numerical method to investigate the coupled diffusion and large deformation problem [13]. Yang and co-workers have employed the large deformation theory to study the diffusion-induced buckling of silicon nanowire electrode [14] and the formulated diffusion equation and the theory of nonlinear elasticity to analyze the influence of local deformation velocity on the evolution of diffusion-induced stress for large deformation electrode [15]. Wen et al. [16] have found that the large deformation and the change of elastic material properties both influence the validity of Stoney-equation for in-situ stress detection in thin film electrode. A variational-based coupled chemomechanical model is established by Zhang et al. [17] to research the phase field fracture and large plastic deformation caused by diffusion for silicon electrode.

One severe consequence from diffusion induced stress is the occurrence of cyclic plasticity behaviour like plastic shakedown or ratcheting, which is thought to be a major factor affecting the mechanical stability and electrochemical performance of battery [18, 19]. Brassart and cooperators [19] have applied a coupled diffusion and finite-strain plasticity theory to study the intercalation-deintercalation cycles of electrode particle and presented the effects of elastic behaviour, elastic shakedown and plastic shakedown on the electrochemical performance of electrode. Haftbaradaran and Gao [20] have investigated the ratcheting behaviour in Si island electrode under cyclic lithiation-delithiation by performing theoretical and numerical calculations. A solution has been presented to obtain the critical size for avoiding the plastic deformation and ratcheting failure. $\mathrm{Xu}$ et al. [21] have provided an analytical model to simulate the ratcheting mechanism of flexible planar Sn electrode considering bending deformation. In another work of this research group [22], the ratcheting behaviour is investigated for both thin film electrode and negative Poisson's ratio electrode. Guarding against the cyclic plasticity accumulation or incremental plastic collapse is crucial in maintaining structural integrity of battery electrode. However, the complexity of cyclic plasticity mechanisms makes it not easily accessible to obtain the analytical solutions. Hence, advanced computational method such as incremental Finite Element Analysis (FEA) is required to model these post yielding behaviour. This step-by-step elastic-plastic method can be applied to investigate the structure response under a combination of various loads, but it will consume enormous computational efforts to produce critical plasticity boundaries like Bree diagram [23].

For efficiently obtaining boundary diagram to avoid alternating plasticity or incremental plastic collapse, a number of direct methods, including the mathematical programming methods [24-26], the Uniform Modified Yield (UMY) surface method [27], the Generalized Local Stress Strain (GLOSS) r-node method [28], the Elastic Compensation Method (ECM) [29] and the Linear Matching Method (LMM) [30, 31], built on the Koiter's [32] kinematic and the Melan's [33] static theorems have been developed and applied in shakedown and ratcheting analyses. In these methods, the LMM has been demonstrated to be capable of providing accurate evaluations on cyclic plasticity behaviour for complex structures under various loading conditions [34, 35], and has also been verified by comparing with experimental tests [36]. Besides, the LMM ABAQUS user subroutines [37] have been solidated by R5 [38] research program of British Energy Generation Ltd. (BEGL) as commercial standard, and widely used in academic research and industrial community. Therefore, the Linear Matching Method is 
adopted in this work to simulate the post yielding behaviour of electrode particle under cyclic electrochemical-mechanical condition.

The objective of this work is to investigate and evaluate the cyclic plasticity failure of primary electrode particle under electrochemical-mechanical condition by utilizing the LMM. With the developed finite element subroutines, the coupled diffusion-stress analysis of electrode particle is conducted for providing the linear elastic solutions for the subsequent cyclic plasticity assessment in the LMM. The shakedown limits and trends of primary particle considering the changing of particle diameter are systematically investigated by using the LMM. ABAQUS step-by-step analysis is adopted to verify the accuracy of the results obtained from the LMM. Low-cycle fatigue damage of electrode particle is evaluated using the calculated plastic strain range from steady state cycle analysis by the LMM. The ratcheting boundaries are also built up so as to avoid the incremental plastic collapse of electrode particle experiencing electrochemical-mechanical load.

\section{Theoretical Background}

\subsection{Diffusion problem}

\subsubsection{Coupled diffusion-stress problem}

The diffusion process obeys the following mass conservation rule $[8,39]$ :

$$
\frac{\partial c}{\partial \tau}+\nabla J=0
$$

Where $c$ and $\tau$ denote concentration and time. $J$ represents diffusion flux. The diffusion flux can be expressed as Eq. (2) in classical Fick's law, where $D$ denotes the diffusion coefficient. The interaction between diffusion and stress generation can be implemented by introducing the stress effect in modifying the diffusion coefficient according to Ref. [40], shown as Eq. (3)

$$
\begin{gathered}
J=-D \nabla c \\
D=D_{0} \exp \left(\frac{\Omega \sigma_{h}}{R T}\right)
\end{gathered}
$$

Where $D_{0}$ is the diffusion coefficient without considering stress effect. $\Omega$ and $\sigma_{\mathrm{h}}$ represent the partial molar volume of Li-ion and the hydrostatic stress. The effect of stress on diffusion is thereby incorporated in updating the diffusion coefficient and diffusion flux. The diffusion boundary condition in the coupled diffusion-stress analysis is set as Neumann boundary condition, as shown below. $i_{n}$ in equation represents the current density acting on the particle surface, and $F_{a}$ is Faraday constant.

$$
J=\frac{i_{n}}{F_{a}}
$$

In the coupled diffusion-stress analysis, due to the fact that the rigidity of bonding material surrounding the particle is far less than that of the particle, it is accessible to make the assumption that there is no traction acting on the particle surface. The mechanical boundary condition can then be given as traction-free surface for single particle having no contact with other particles and shown as Eq. (5) [8], where $l, m, n$ are direction cosines between axis and external normal. It is worth noting that the Eq. (5) does not apply for multiple particles contacting each other.

$$
\begin{aligned}
& F_{s x}=\sigma_{x} l+\tau_{x y} m+\tau_{x z} n=0 \\
& F_{s y}=\tau_{y x} l+\sigma_{y} m+\tau_{y z} n=0 \\
& F_{s z}=\tau_{z x} l+\tau_{z y} m+\sigma_{z} n=0
\end{aligned}
$$




\subsubsection{Finite element implementation}

For implementing the diffusion-stress coupling in finite element software ABAQUS, one can take the diffusion behaviour analogous to the heat conduction behaviour [41]. The heat conduction process follows the energy conservation law:

$$
\rho c_{s} \frac{\partial t}{\partial \tau}+\nabla q=\varnothing
$$

$\rho, c_{S}, \emptyset$ are density, specific heat, internal heat source. $t$ and $q$ are temperature and heat flux respectively. It is available to analogize mass diffusion with heat conduction by using the following hypothesis.

$$
\begin{array}{ll}
t=c & \varnothing=0 \\
q=J & \rho c_{s}=1
\end{array}
$$

Additionally, the thermal expansion coefficient can be replaced by partial molar volume with $\alpha=\frac{\Omega}{3}$ [8]. The aforementioned formulas make it available for converting ABAQUS coupled thermal-stress analysis to coupled diffusion-stress analysis with developing UMATHT subroutine [42].

\subsection{The framework of Linear Matching Method}

In this work, we consider a structure experiencing a cyclic history of diffusion-induced stress $\lambda_{d} D(x, t)$ throughout its volume $V$ and cyclic surface load $\lambda_{p} P(x, t)$ acting on portion of the structure surface $S_{T}$. $\lambda$ herein represents a scalar load multiplier and the rest part of structure surface $S_{u}\left(S=S_{T}+S_{u}\right)$ is fixed. One loading cycle is assumed to be in the time interval $0 \leq t \leq \Delta t$. The material of the structure is set as isotropic, elastic-perfectly plastic and obeys von Mises yielding condition. The linearelastic solution of the aforementioned loading condition can be given as

$$
\hat{\sigma}_{i j}(x, t)=\lambda_{d} \hat{\sigma}_{i j}^{d}(x, t)+\lambda_{p} \hat{\sigma}_{i j}^{P}(x, t)
$$

$\hat{\sigma}_{i j}^{d}(x, t)$ and $\hat{\sigma}_{i j}^{P}(x, t)$ represent the linear-elastic solutions produced by $D(x, t)$ and $P(x, t)$ respectively.

After plenty of cycles, the stresses and strain rates will approximate to a cyclic state, i.e.,

$$
\sigma_{i j}(t)=\sigma_{i j}(t+\Delta t), \quad \dot{\varepsilon}_{i j}(t)=\dot{\varepsilon}_{i j}(t+\Delta t)
$$

The cyclic stress history herein consists of three components,

$$
\sigma_{i j}(x, t)=\lambda \hat{\sigma}_{i j}(x, t)+\bar{\rho}_{i j}(x)+\rho_{i j}^{r}(x, t)
$$

Where $\lambda \hat{\sigma}_{i j}$ represents the elastic stress solution, $\bar{\rho}_{i j}$ is a constant residual stress field in equilibrium with no surface traction acting on $S_{T}$ and corresponds to the residual stress filed at the start and end of the cycle. $\rho_{i j}^{r}$ denotes the variation of residual stress within the cycle and follows

$$
\rho_{i j}^{r}(x, 0)=\rho_{i j}^{r}(x, \Delta t)
$$

\subsubsection{Evaluation of the shakedown limit}

The shakedown analysis in the LMM calculates both the upper and lower bounds of shakedown limit using the Koiter's upper bound theorem and Melan's lower bound theorem respectively. Koiter's theorem presents that the shakedown behaviour will not happen if any kinematically admissible strain rate can be satisfied such that the strain field is compatible with the applied displacements and the plastic dissipation is less than or equal to the applied work. Melan states that the 
structure will enter shakedown state if there is a constant residual stress field such that, for any combination of residual stress filed and elastic stress field caused by the applied load, the yielding criterion is not violated within the whole structure.

The cyclic stress history in shakedown problem can be simplified to the following form, as the variation of residual stress $\rho_{i j}^{r}$ equals to zero.

$$
\sigma_{i j}(x, t)=\lambda \hat{\sigma}_{i j}(x, t)+\bar{\rho}_{i j}(x)
$$

The upper bound shakedown limit $\lambda_{U B}^{S}$ can be obtained through

$$
\lambda_{U B}^{S}=\frac{\int_{V} \int_{0}^{\Delta t} \sigma_{y} \overline{\dot{\varepsilon}}\left(\dot{\varepsilon}_{i j}\right) d t d V}{\int_{V} \int_{0}^{\Delta t}\left(\widehat{\sigma}_{i j} \dot{\varepsilon}_{i j}\right) d t d V}
$$

Where $\sigma_{y}$ is the yield strength of the material, $\overline{\dot{\varepsilon}}$ and $\dot{\varepsilon}_{i j}$ represent the effective strain rate and kinematically admissible strain rate. In shakedown problem, the combination of the elastic stress fields and constant residual stress field under von Mises yielding condition will not lead to the accumulation of plastic strain. It is thereby to obtain the lower bound of shakedown limit through applying the following equation at all integration points for each loading instances.

$$
f\left(\lambda_{L B}^{S} \hat{\sigma}_{i j}\left(x_{i}, t\right)+\bar{\rho}_{i j}\left(x_{i}\right)\right) \leq 0
$$

$\lambda_{L B}^{S}$ in equation denotes the multiplier of lower bound shakedown limit.

\subsubsection{Evaluation of the ratcheting limit}

The evaluation of ratcheting limit can be decomposed to two parts [43]. The first part is to solve the varying residual stress field $\rho_{i j}^{r}$ which is implemented as the Direct Steady Cycle Analysis (DSCA) of LMM framework. The DSCA involves $m$ iterative cycles, where $m=1,2 \cdots M$. Each iterative cycle consists of $n$ increments, where $n=1,2 \cdots N$ for $N$ load instances. By employing Eq. 15 , it is available to calculate the constant residual stress $\bar{\rho}_{i j}^{r}$.

$$
\bar{\rho}_{i j}^{r}=\sum_{m-1}^{M} \sum_{n-1}^{N} \Delta \rho_{i j}^{r}\left(x_{k}, t_{n}\right)_{m}
$$

where $\Delta \rho_{i j}^{r}\left(x_{k}, t_{n}\right)_{m}$ denotes the changing residual stress corresponding to elastic stress $\sigma_{i j}^{\Delta}\left(x_{k}, t_{n}\right)$. The varying residual stress $\rho_{i j}^{r}\left(x, t_{n}\right)$ at the steady state cycle can then be given as

$$
\rho_{i j}^{r}\left(x, t_{n}\right)=\bar{\rho}_{i j}^{r}(x)+\sum_{k=1}^{n} \Delta \rho_{i j}^{r}\left(x, t_{k}\right)_{M}
$$

The associated converged increment of plastic strain at time $t_{n}$ is described as

$$
\Delta \varepsilon_{i j}^{p}\left(x, t_{n}\right)=\frac{1}{2 \bar{\mu}_{n}\left(x, t_{n}\right)}\left[\hat{\sigma}_{i j}^{\Delta^{\prime}}\left(x, t_{n}\right)+\rho_{i j}^{r^{\prime}}\left(x, t_{n}\right)\right]
$$

$\bar{\mu}_{n}$ in equation denotes the iterative shear modulus which can be calculated by linear matching equation [43]. $\hat{\sigma}_{i j}^{\Delta}$ is the linear elastic solution of the predefined cyclic loading and the notation (') represents the deviator component of the stress and strain.

The second part is to apply the shakedown theorem to calculate the ratcheting limit, where the linear elastic solution of the predefined cyclic loading is modified by the changing residual stress $\rho_{i j}^{r}\left(x, t_{n}\right)$. By using the von Mises yielding criteria, an upper bound multiplier of ratchet limit can be given as

$$
\lambda=\frac{\int_{V} \sum_{n=1}^{N}\left(\sigma_{y} \bar{\varepsilon}\left(\Delta \varepsilon_{i j}^{n}\left(x, t_{n}\right)\right)\right) d V-\int_{V} \sum_{n=1}^{N}\left(\left(\widehat{\sigma}_{i j}^{\Delta}\left(x, t_{n}\right)+\rho_{i j}^{r}\left(x, t_{n}\right)\right) \Delta \varepsilon_{i j}^{n}\left(x, t_{n}\right)\right) d V}{\int_{V} \widehat{\sigma}_{i j}^{\bar{F}}(x)\left(\sum_{n=1}^{N} \Delta \varepsilon_{i j}^{n}\left(x, t_{n}\right)\right) d V}
$$

Where $\bar{\varepsilon}\left(\Delta \varepsilon_{i j}^{n}\right)=\sqrt{\frac{2}{3} \Delta \varepsilon_{i j}^{n} \Delta \varepsilon_{i j}^{n}}$ and $\Delta \varepsilon_{i j}^{n}$ denotes a kinematically admissible plastic strain rate. The ratcheting multiplier $\lambda$ here defines the capacity of the structure subjected to a combination of cyclic 
loads to accommodate an additional constant load without ratcheting happens. According to the aforementioned framework, the LMM iteratively generates a series of monotonically decreasing upper bounds and converges to the least upper ratchet limit for the selected class of displacement fields. The aforementioned numerical methods are implemented in ABAQUS [42] by developing user subroutines and detailedly described in Ref. [43].

\section{Problem Description}

\subsection{Finite element model and material property}

The model of primary electrode particle is constructed and meshed by employing the finite element program ABAQUS. The geometry and mesh configurations are identical in the coupled diffusion-stress analysis and cyclic plasticity analysis. For element type, C3D20RT is employed for coupled diffusionstress analysis and C3D20R is applied in cyclic plasticity analysis.

The adopted properties in numerical work for the NCM material are listed in Table 1. The NCM material is assumed as isotropic, elastic-perfectly plastic and obeys von Mises yielding condition in this work. The elastic-perfectly plastic constitutive equation $\left(\sigma=E \varepsilon, \varepsilon \leq \varepsilon_{y} ; \sigma=E \varepsilon_{y}, \varepsilon>\epsilon_{y}\right)$ is commonly applied to model the stress-strain response for electrode material $[47,48]$, and is accessible to simulate the mechanical behaviour of the material in the lack of hardening material properties. Considering the hardening effect is not significant for NCM electrode, the material is assumed to follow the elastic-perfectly plastic law in this work. The yield stress of NCM material is to be considered within a range, and it is determined by material preparation, material composition, etc. The preparation technology for such an oxide electrode material makes it difficult to experimentally measure the exact value of the yield strength, which is also rarely reported in the literature. The widely used yield stress $100 \mathrm{MPa}$ for oxide electrode material having similar lattice structure with NCM is employed in this paper.

According to the section 2.1.2, the diffusion-induced expansion strain can be calculated as $\frac{\Omega}{3} \Delta c[8,49]$. For NCM electrode material, the maximum expansion strain is $3.3761 \%$. Based on the Hooke's law, the maximum elastic strain can be obtained as $0.08 \%$. The total strain is less than $4 \%$ for NCM material, which makes it reasonable to consider the material satisfying small deformation assumption.

Table 1. Material properties of NCM electrode

\begin{tabular}{ll}
\hline Property & Value \\
\hline Young's modulus $(\mathrm{GPa})$ & $125[44]$ \\
Poisson ratio & $0.3[44]$ \\
Maximum concentration $\left(\mathrm{mol} / \mathrm{m}^{3}\right)$ & $48230[45]$ \\
Diffusivity $\left(\mathrm{cm}^{2} / \mathrm{s}\right)$ & $10^{-11[45]}$ \\
Partial molar volume $\left(\mathrm{m}^{3} / \mathrm{mol}\right)$ & $2.1 \mathrm{E}-6[46]$ \\
Yield strength $(\mathrm{MPa})$ & 100 \\
\hline
\end{tabular}

\subsection{Linear Elastic Solutions}

The theoretical foundation of the Linear Matching Method can be summarized as utilizing a series of iterative linear analyses to model the nonlinear material response, and represent the history of stress and inelastic strains. Therefore, calculating the linear elastic solutions of electrode particle under lithiation-delithiation condition works as the first step before performing the subsequent cyclic plasticity assessment.

By employing ABAQUS with the developed user subroutines, it is available to investigate the concurrent diffusion and stress generation process for spherical particle during intercalation- 
deintercalation condition. Fig. 1 shows the history of cyclic diffusion-induced stress for 3um diameter electrode particle under $0.3 \mathrm{~A} / \mathrm{m}^{2}$ within one cycle. The DoD and SoC on X-axis respectively denote the depth of discharge and the state of charge, which are used to describe the status of electrode particle in the lithiation-delithiation cycle. Due to the variation of concentration gradient caused by Liion flow, the diffusion-induced stress presents the initial decreasing and subsequent increasing trends in both lithiation and delithiation phases. The LMM applies finite representative loading instants to describe the entire loading history and the rest instants with smaller loads are assumed to be encompassed. According to Fig. 1, four instants representing extreme situations are employed to reflect the cyclic diffusion-induced stress history. The contours of stress and concentration fields at these four instants are presented in Fig. 2 (a) and (b) respectively. Instant 1 is the time point at the start of lithiation process (discharge phase, DoD $0 \%$ ), when the residual concentration gradient from last working cycle results in the residual stress field. Due to the influx of Li-ion, the concentration gradient is rapidly reduced leading to the decrease of diffusion-induced stress, and the instant with the minimum stress is defined as instant 2 at lithiation phase (discharge phase, DoD 0.84\%). Hereafter, the continuing lithiation makes the concentration gradient reversely magnified, leading to the rise of diffusion-induced stress to instant 3 (discharge phase, DoD 100\%), which experiences the maximum stress in lithiation process. For the delithiation process, it also experiences the initial decrease phase and the subsequent increase phase of concentration gradient and stress field due to the outflow of Liion. The inflection point in this process is presented as instant 4 (charge phase, SoC $0.84 \%$ ) with the minimum stress. Hence, the instants with the maximum and minimum stresses in both lithiation and delithiation phases are applied to construct the loading cycle.

Besides, the lithiation caused swelling will result in the extrusion force between neighbouring particles as illustrated in Fig. 2 (c), which will further influence the carrying capacity of the configuration. Fig. 1 also gives the variation of a mechanical reference stress on electrode particle within lithiationdelithiation cycle, where the extremum of the mechanical stress emerges at the end moment of intercalation process when the configuration is assumed to experience the maximum expansion and is defined as instant 3 (discharge phase, DoD 100\%). The contour of mechanical reference stress at instant 3 is given as Fig. 2 (d). This varying mechanical reference stress is applied as the reference load for the LMM and is scaled to represent the cyclic plasticity limit of the configuration.

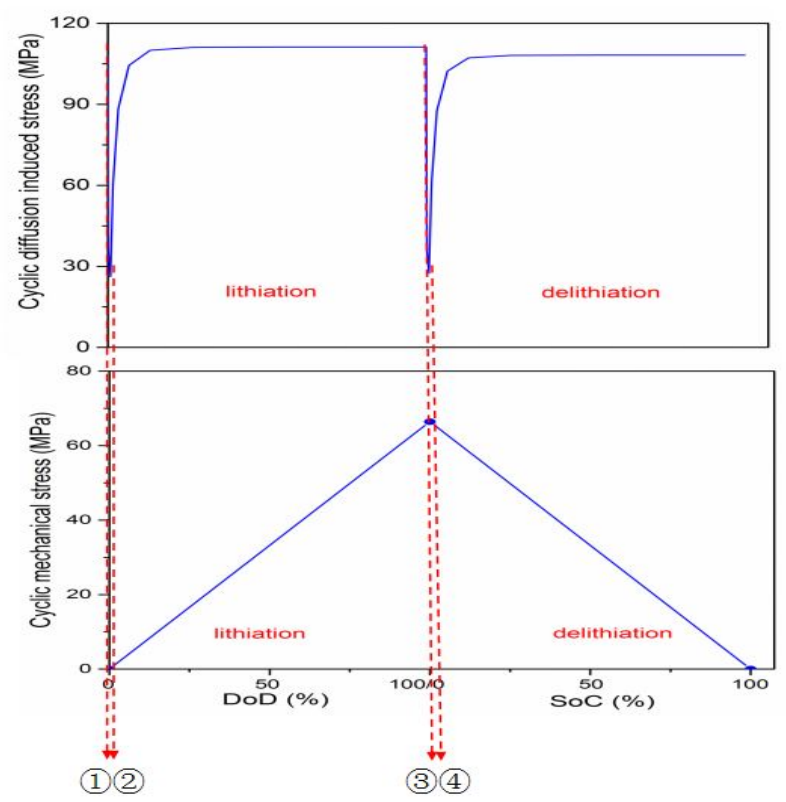

Fig. 1 The history of diffusion-induced stress under $0.3 \mathrm{~A} / \mathrm{m}^{2}$ and mechanical reference stress for 3um diameter electrode particle within one cycle (DOD - depth of discharge, SOC - state of charge) 


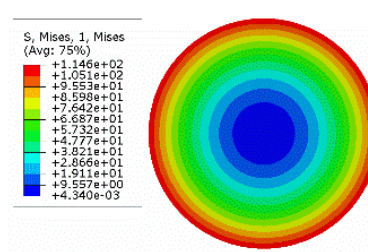

lithiation DoD 0\% (instant 1)

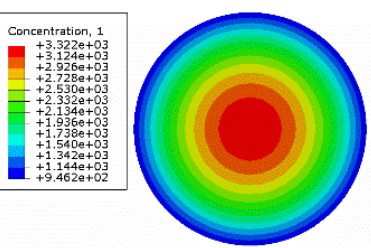

lithiation DoD 0\% (instant 1)

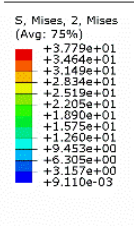

lithiation DoD $0.84 \%$ (instant 2)

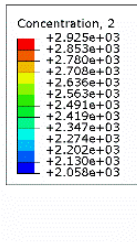

lithiation $\mathrm{DoD} 0.84 \%$ (instant 2 )

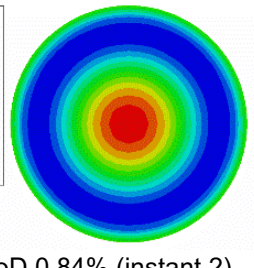

(b)

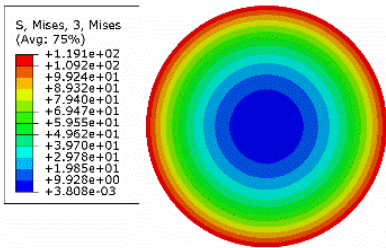

(a)

a)
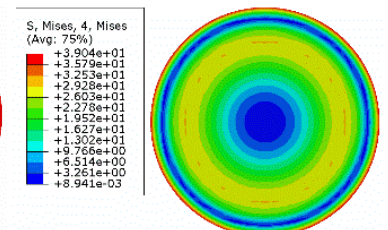

delithiation SoC $0.84 \%$ (instant 4 )
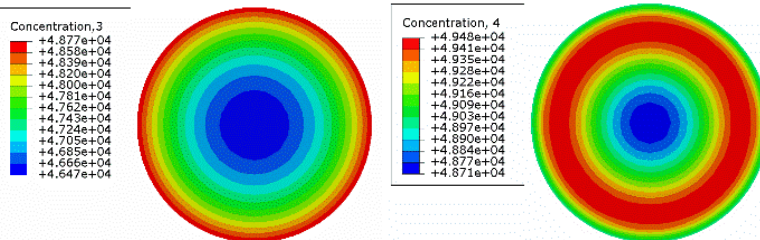

delithiation SoC $0.84 \%$ (instant 4 )

(b)

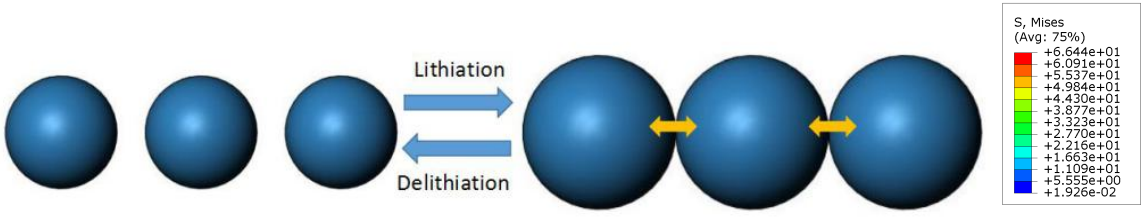

(c)

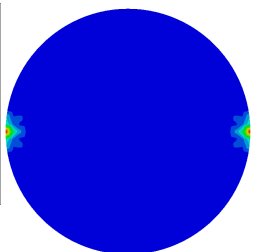

(d)

Fig. 2 (a) The contours of elastic von Mises effective stress of four selected instants, (b) the contours of concentration fields of four selected instants, (c) the schematic diagram of lithiation caused extrusion between adjacent particles, (d) the contour of mechanical reference stress at instant 3

\section{Shakedown Assessment}

The shakedown limit in this section is defined as the capacity to withstand cyclic current density leading to the generation of diffusion-induced stress and cyclic mechanical load. The LMM is employed to perform shakedown analysis and ABAQUS step-by-step inelastic assessment is also conducted so as to testify the accuracy of the acquired shakedown boundary from LMM.

\subsection{Shakedown limits of electrode particle}

By calculating different combinations of cyclic current density and mechanical load, it is available to obtain the shakedown limit curves for electrode particle subjected to cyclic lithiaion-delithiation process. Fig. 3 (a) presents the shakedown boundaries for a primary electrode particle with $3 \mathrm{um}$ diameter under the cyclic current density and symmetrically isometrical external pressures caused by adjacent electrode particles. It is observable that the loading range is separated by shakedown boundary to two parts as shakedown zone and cyclic plasticity zone. The Y-coordinate in Fig. 3 (a) denotes the strength of current density which leads to the generation of cyclic diffusion-induced stress, and X-coordinate in Fig. 3 (a) represents the ratio between the applied mechanical load and mechanical reference load. Additionally, the loading conditions on Y-axis of Fig. 3 (a) represent the shakedown limits of primary particle subjected to only cyclic diffusion-induced stress, where the particle is presumed to have no contact with neighbouring particles when swelling happens.

It should be highlighted that the different particle arrangements such as schematic diagrams shown in Fig. 3 (b) and (c) may also affect the cyclic plasticity limit of electrode particle. In Fig. 3 (b), the central particle will withstand two symmetric external pressures during swelling process, and the central particle in Fig. 3 (c) will experience four symmetric external pressures accompanying with lithiation phase. The cyclic plasticity boundaries for these two scenarios are presented in Fig. 3 (a), 
and it is noteworthy that the shakedown boundaries under two symmetric external pressures are identical with that under four symmetric external pressures. Fig. 4 (a) and (b) provide the elastic von Mises stress solutions for particle under two and four mechanical reference loads. It is observed that two extra mechanical loads have no impact on the maximum von Mises stress which always emerges at where the mechanical load acts. In addition, it is known that the shakedown limit is decided by the maximum elastic stress range. Hence, the shakedown limits for particle under two or four isometrical external pressures are identical.

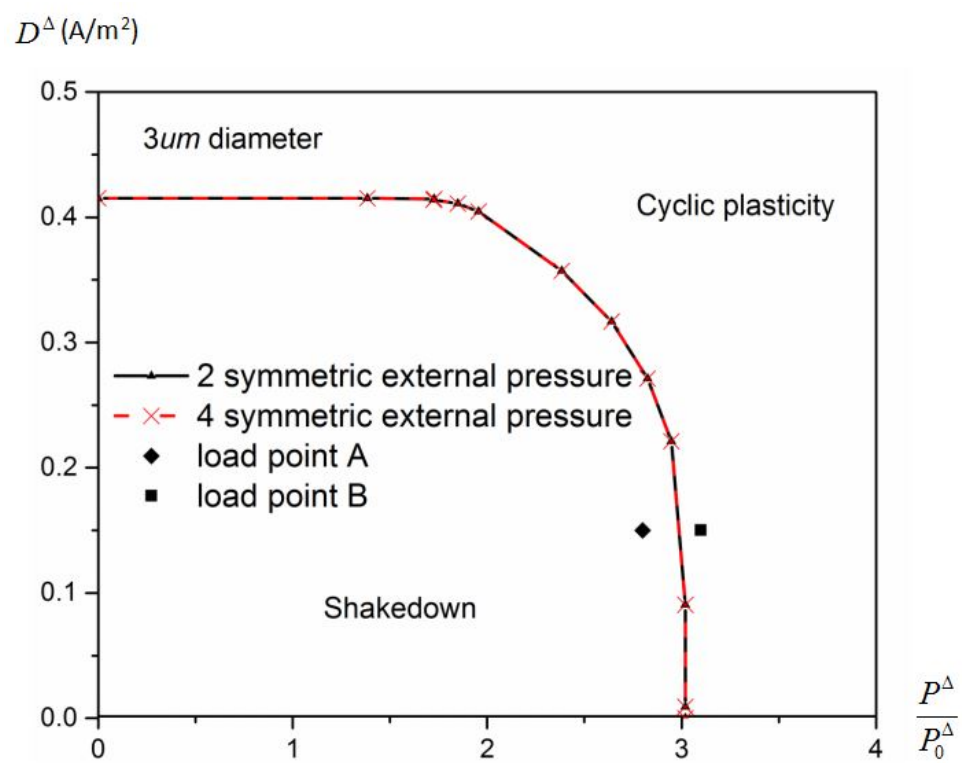

(a)

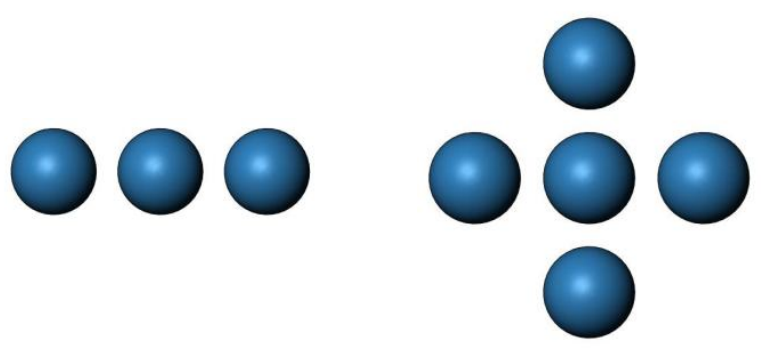

(b)

(c)

Fig. 3 (a) Shakedown boundaries of 3um diameter particle under cyclic current density and mechanical load considering different particle arrangements, the schematic diagrams of different particle arrangements, (b) one central particle with two neighbouring particles, (c) one central particle with four neighbouring particles.

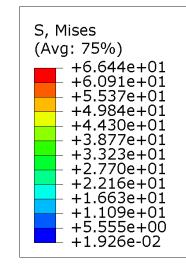

(a)

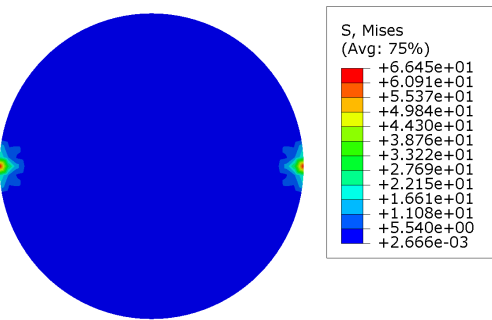

(a)

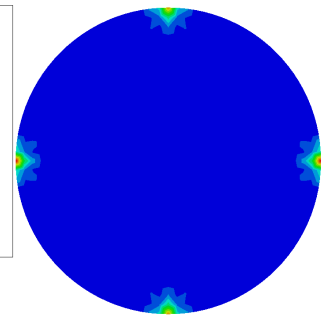

(b)

Fig. 4 The distribution of von Mises stress for 3um diameter particle under (a) two, and (b) four mechanical reference loads 


\subsection{ABAQUS step-by-step analysis}

For validating the accuracy of the obtained shakedown limits from the LMM, loading conditions in and out of the shakedown boundary are chosen for conducting the ABAQUS step-by-step analyses. Fig. 5 (a) and (b) present the plastic strain history (PEMAG) for the most dangerous position of the particle under load points A and B shown in Fig. 3 (a). The evolution of plastic strain in Fig. 5 (a) presents that there will be no more new plastic strain generated after early cycles, which indicates the particle will experience shakedown mechanism subjected to load point A. It is observed from Fig. 5 (b) that, the produced positive plastic strain is equal to the negative plastic strain for the particle under the load point $B$ at the steady state, which shows the reverse plasticity status of the structure. Therefore, the applicability of employing LMM to perform the cyclic plasticity evaluation for electrode particle has been verified via ABAQUS step-by-step analysis.

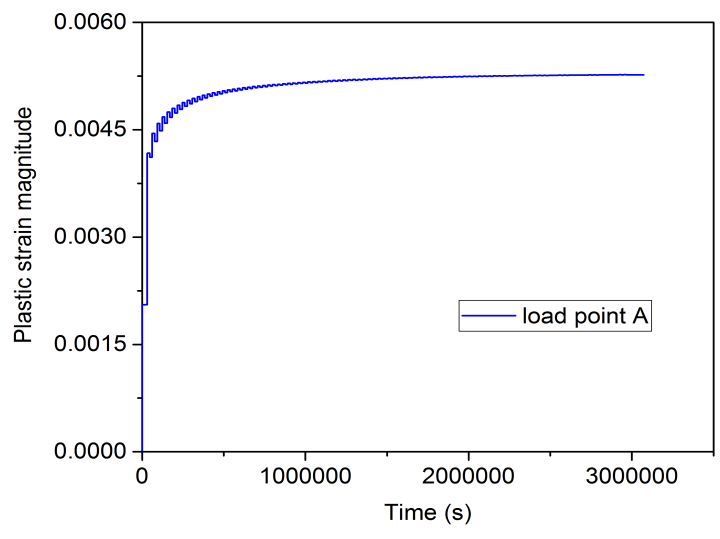

(a)

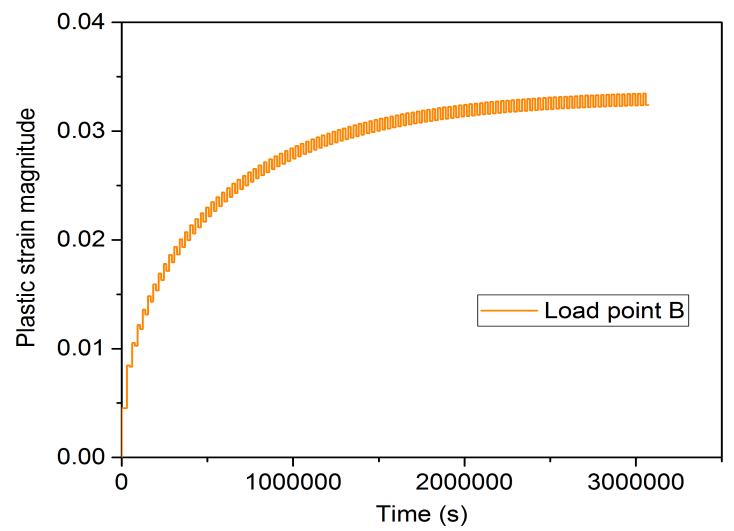

(b)

Fig. 5 Plastic strain history (PEMAG) obtained from ABAQUS step-by-step analyses for cyclic loading (a) load point A, (b) load point B

\subsection{Shakedown boundaries with the changing of particle diameter}

Due to the non-uniform size distribution of active material particles in micrometer scale, it is significant to investigate how the size variation affects the shakedown boundaries of the particle. Fig. 6 presents the shakedown boundaries for electrode particle with varying dimensions subjected to cyclic current density and mechanical load. The shakedown limits on $\mathrm{Y}$-axis present the permissible current density for individual particle losing contact with adjacent particles during swelling process. It is observed as general trend that this permissible current density decreases with an increase of particle dimension. In our previous work [12], we have also concluded the similar phenomenon that, with just considering the effect from current density, the particle with larger size is more vulnerable to initiate crack and proceed crack propagation. Fig. 7 (a) and (b) show the maximum von Mises stress during the lithiation-delithiation process for particles with diameter 2 um and 4 um only under $0.15 \mathrm{~A} / \mathrm{m}^{2}$. It can be seen that the stress generated of 2 um diameter particle is less than that of the particle with 4um diameter under the same electrochemical condition, thus the smaller particle is capable of withstanding greater current density. 


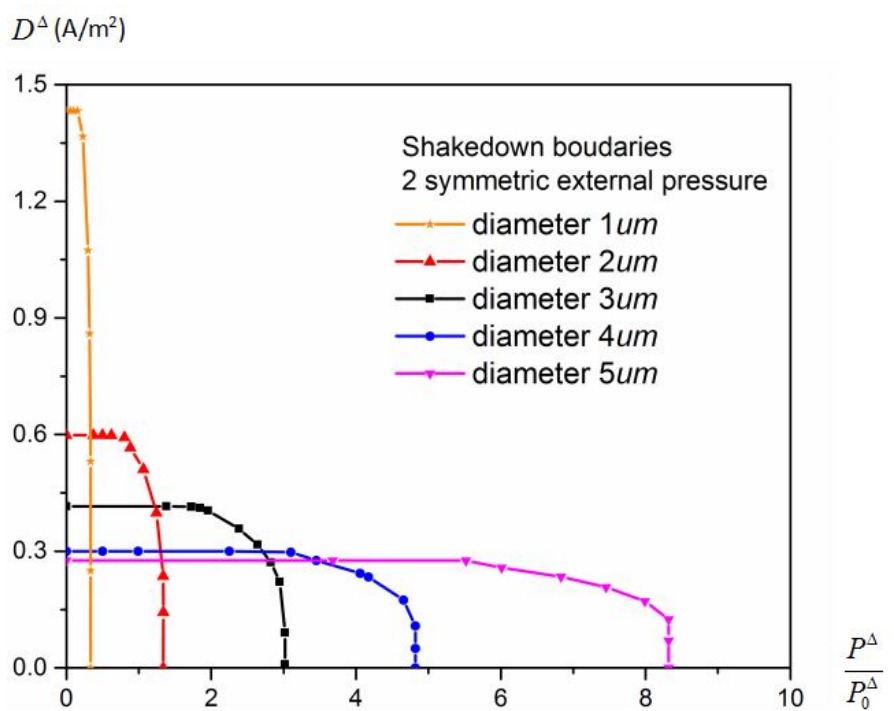

Fig. 6 The shakedown limit curves of the electrode particle with various dimensions subjected to cyclic current density and mechanical load

Another notable point is that, under the same current density, the capacity for particle to withstand mechanical load is in positive correlation with the increasing of particle dimension. Fig. 7 (c) and (d) illustrate the maximum von Mises stress for particles with diameter 2um and 4um only under mechanical reference load. In contrast to the electrochemical load, the particle with smaller size will experience relatively greater stress than that of the larger particle under the same mechanical load, which makes the larger particle accessible to withstand more mechanical load. The aforementioned mechanisms decide the changing trend of shakedown boundaries for particles with varying dimensions, and the change of particle size makes considerable impact on the carrying capacity of electrode particle under electrochemical-mechanical condition.

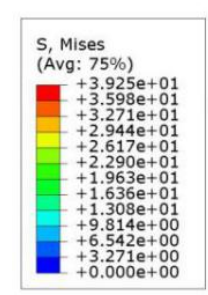

(a)

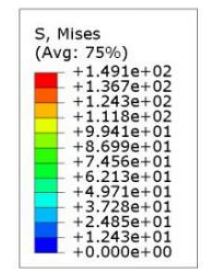

(c)
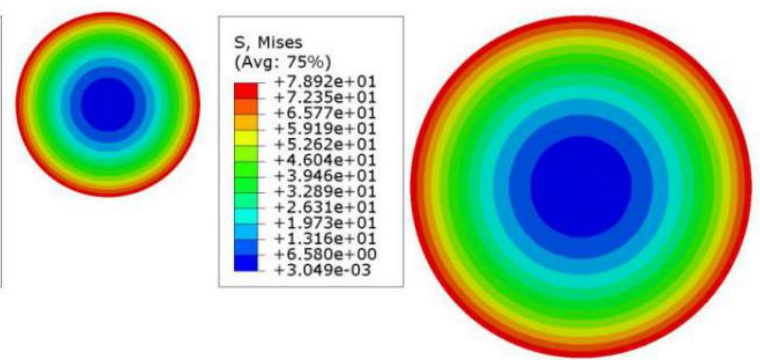

(b)
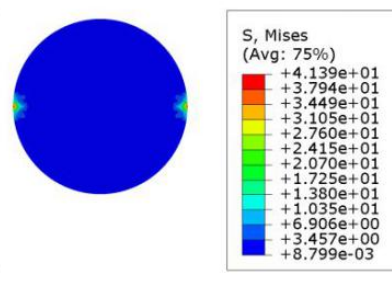

(d)

Fig. 7 The maximum von Mises stress under $0.15 \mathrm{~A} / \mathrm{m}^{2}$ (a) particle with diameter $2 \mathrm{um}$, (b) particle with diameter $4 u m$, the maximum von Mises stress under mechanical reference load (c) particle with diameter $2 \mathrm{um}$, (d) particle diameter 4um 


\section{Steady state cycle and ratchet limit analysis}

Two categories of failure mechanisms will occur when the configuration subjects to the loading condition out of shakedown boundary. In a loading cycle at steady state, when the produced plastic strain forms a closed cycle, the structure is assumed to be in reverse plasticity status. Associating the generated plastic strain range with material fatigue parameters, it is available to assess the low-cycle fatigue damage for the structure. Alternatively, if the plastic strain settles into an open cycle, the ratcheting behaviour is considered to happen where the strain accumulation will lead to the incremental plastic collapse of the structure.

\subsection{Steady state cycle analysis}

By employing the direct steady cycle analysis in the LMM, it is accessible to efficiently obtain the plastic strain range of the structure under certain loading condition and further evaluate the corresponding low-cycle fatigue damage. Fig. 8 (a) presents the shakedown boundary of 3um particle with three loading histories out of shakedown boundary. The locations of these three loading points are relatively far from the shakedown boundary so as to present more obvious plastic strain range and low-cycle fatigue damage. Fig. 8 (b), (c) and (d) illustrate the corresponding contours of plastic strain range for 3um particle under these loading histories, where the maximum values of plastic strain range reach $0.09905,0.03395$ and 0.001335 respectively. For loading point 1 and 2, the plastic strain range is mainly dominated by the cyclic mechanical load, where the significant plastic strain range appears to occur at the area applied with mechanical load. Whereas for loading point 3 , the cyclic diffusioninduced stress works as the primary effective load. The maximum plastic strain range locates at the outermost layer of the particle where the maximum diffusion-induced stress happens.

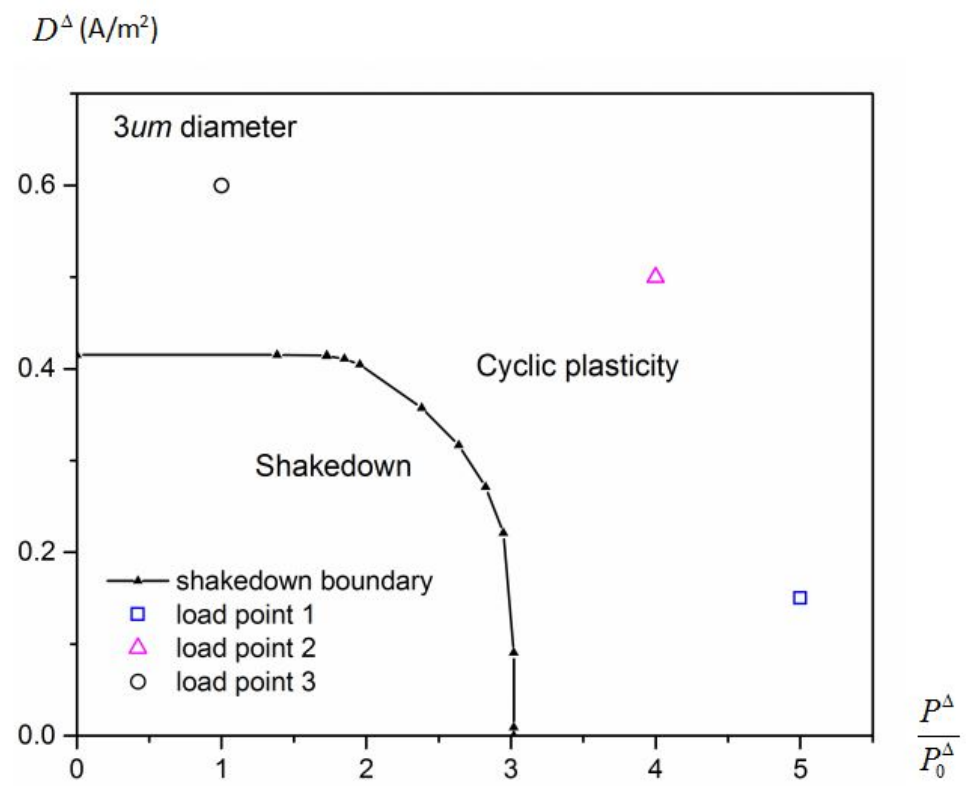

(a)

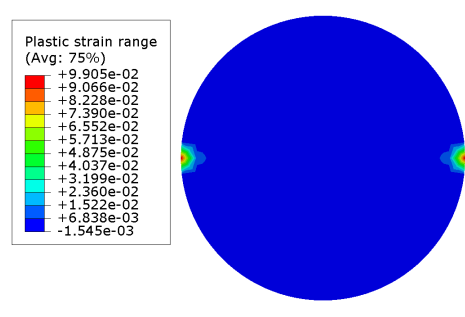

(b) loading point 1
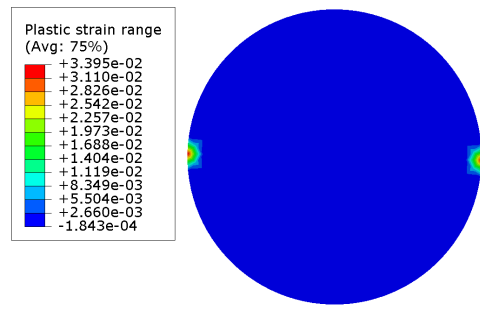

(c) loading point 2
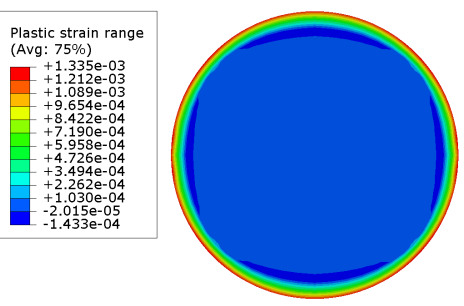

(d) loading point 3 

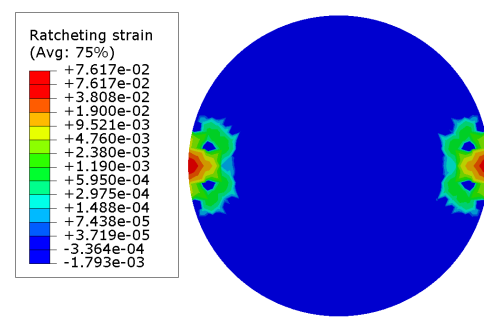

(e) loading point 1
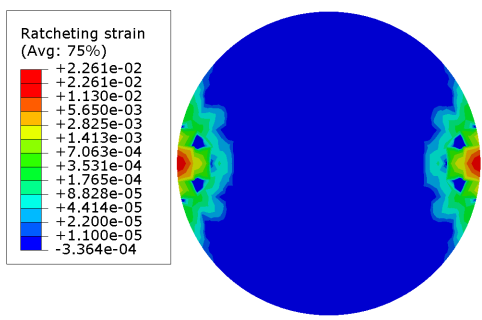

(f) loading point 2
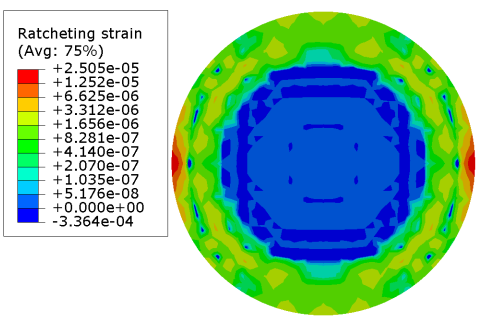

(g) loading point 3

Fig. 8 (a) shakedown boundary of 3um particle with selected loading points, the contours of plastic strain range of 3 um particle under (b) loading point 1, (c) loading point 2, (d) loading point 3, the contours of ratcheting strain of 3 um particle under (e) loading point 1 , (f) loading point 2 , (g) loading point 3

According to the Manson-coffin equation shown below, the plastic strain range can be used as the input parameter to predict the fatigue life.

$$
\frac{\Delta \varepsilon_{p}}{2}=\varepsilon_{f}^{\prime}(2 N)^{c^{\prime}}
$$

where $\Delta \varepsilon_{p}$ is the plastic strain range, $2 \mathrm{~N}$ denotes the number of reversals to failure. $\varepsilon_{f}$ and $c^{\prime}$ represent two empirical constants known as the fatigue ductility coefficient and the fatigue ductility exponent respectively. By formula transformation, it is available to obtain the caused fatigue damage per cycle at steady state.

$$
2\left(\frac{2 \varepsilon_{f}^{\prime}}{\Delta \varepsilon_{p}}\right) \frac{1}{c^{\prime}}=\frac{1}{N_{i}}=d D_{m i}
$$

Table 2 presents the fatigue ductility coefficient and fatigue ductility exponent for NCM ternary material [50]. According to the generated plastic strain range, it is thereby available to obtain the corresponding low-cycle fatigue damage per cycle of electrode particle at steady state. Table 3 shows the fatigue damage per cycle of the most dangerous position on particle and the corresponding number of cycles to low-cycle fatigue failure under three loading histories. It is obvious that, under different loading conditions, the generated fatigue damage varies greatly. For loading points 1 and 2, the generated low-cycle fatigue damage makes a relatively great contribution to the structural failure. While for the loading point 3, the low-cycle fatigue caused damage is not significant. Additionally, the ratcheting strain can also be given by using the direct steady cycle analysis, as shown in Fig 8 , where the ratcheting strain is defined as the net accumulation of the plastic strain per cycle. It is available to see that, there is relatively small ratcheting strain generated under loading point 3 . While for the loading points 1 and 2 , the obvious ratcheting strain will accumulate at the mechanical load acting area and the ratcheting failure assessment is required to conduct.

Table 2. Fitted parameters in the fatigue damage model

\begin{tabular}{ll}
\hline$\varepsilon_{f}^{\prime}$ & $c^{\prime}$ \\
\hline 3.184 & -0.688 \\
\hline
\end{tabular}

Table 3. Fatigue damage and number of cycles to fatigue failure

\begin{tabular}{lll}
\hline & Maximum damage per cycle & Number of cycles to failure \\
\hline Load point 1 & $4.7 \mathrm{e}-3$ & 212 \\
Load point 2 & $9.9 \mathrm{e}-4$ & 1010 \\
Load point 3 & $9.0 \mathrm{e}-6$ & 111111 \\
\hline
\end{tabular}




\subsection{Ratcheting assessment}

A body experiencing ratcheting behaviour, which eventually results in the incremental plastic collapse, should be rigorously avoided. The ratcheting limit in this work is defined as the capacity for configuration under a predefined cyclic load to accommodate an extra constant load without representing ratcheting failure.

With the calculation of different combinations of cyclic current density and constant mechanical load, we plot the ratcheting boundaries for 3um particle shown in Fig. 9, where the shakedown boundaries are also given. In this case, the ratcheting limit can be expressed as the capacity for structure experiencing cyclic current density to withstand extra constant mechanical load. For the $Y$-axis, the arbitrary applied cyclic current density is normalized with respect to a reference current density set as $0.3 \mathrm{~A} / \mathrm{m}^{2}$. The entire loading range is divided by boundaries to three zones known as shakedown zone, reverse plasticity zone and ratcheting zone, which makes it easily accessible to understand the structural state under certain loading condition. The contour plot in Fig 9 presents the ratcheting behaviour caused failure state of electrode particle, which indicates that the strain accumulations are mainly centered on the mechanical loading acting area.

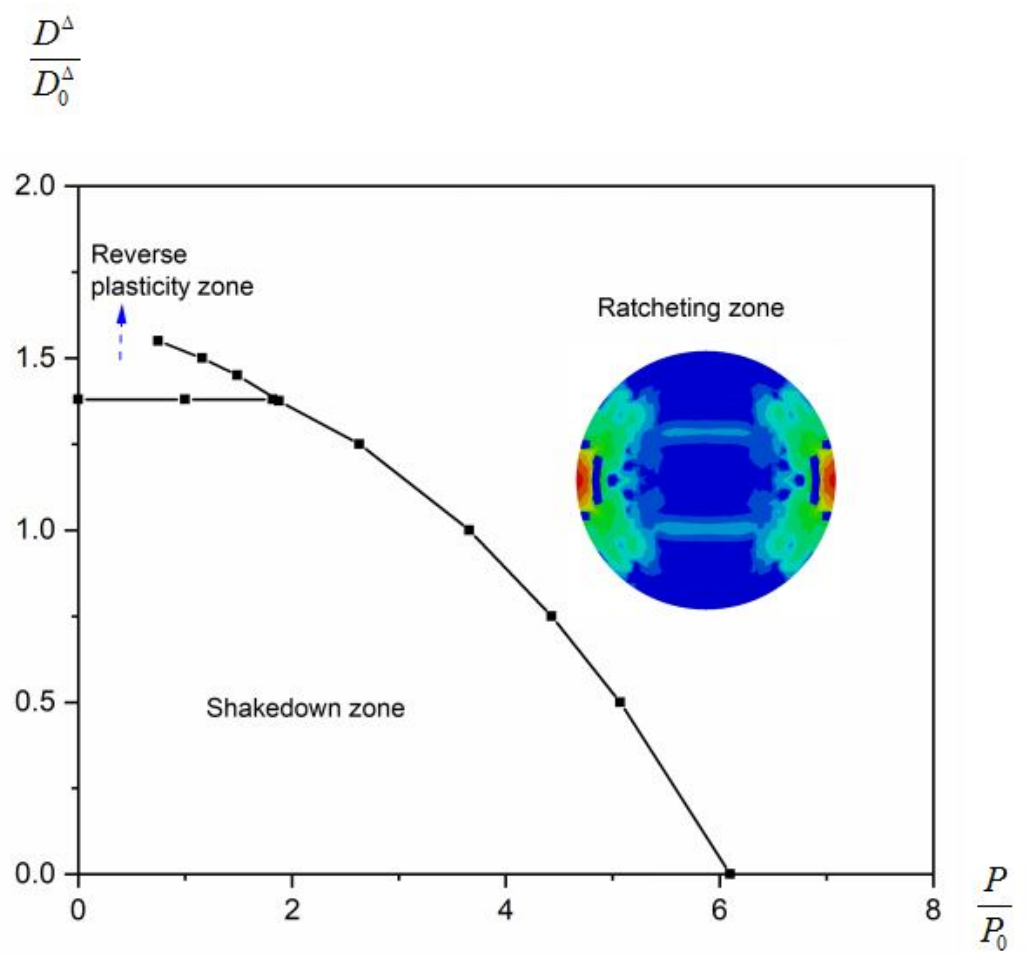

Fig. 9 Ratcheting and shakedown boundaries for 3um particle subjected to cyclic current density and constant mechanical load

Considering the variation of particle diameter, we present the ratcheting boundaries for electrode particle with various dimensions under cyclic current density and constant mechanical load in Fig. 10. As explained in shakedown analysis, it is concluded that the capacity for particle to withstand electrochemical load decreases with an increase in the particle size. On the contrary, larger particle is able to bear more mechanical load. Hence, the ratcheting boundaries for electrode particles with various dimensions evolve in this way. 


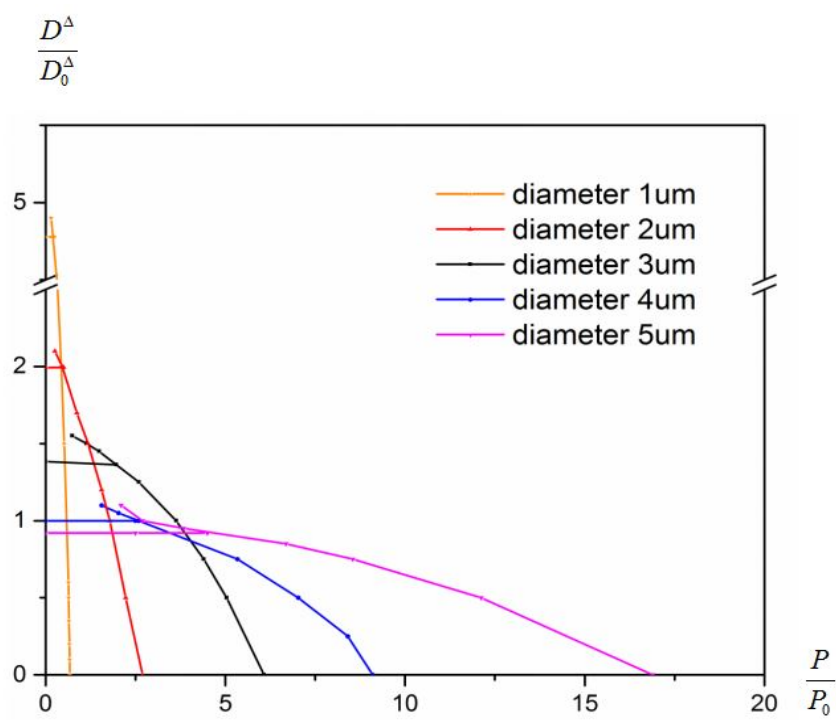

Fig. 10 Ratcheting and shakedown limit curves for electrode particle with various dimensions under cyclic current density and constant mechanical load

For comprehensively investigating the carrying capacity of electrode particle under electrochemicalmechanical load, we present the ratcheting and shakedown limit curves in another way shown in Fig. 11, where the cyclic plasticity limit is expressed as the particle subjected to a coupled cyclic current density - mechanical load $P D^{\Delta}$ to withstand an additional constant mechanical load $P$. The values on $Y$-axis represent the ratio between the applied coupled current density - mechanical load and coupled reference current density - mechanical load. As the cyclic plasticity carrying capacity of small particle is sensitive to mechanical load, both the shakedown and ratcheting limits of 1um and $2 \mathrm{um}$ particles are greatly reduced with the adding of cyclic mechanical load to the predefined loading conditions. On the contrary, the cyclic plasticity limits of bulky particles (diameter 4um and 5um) are rarely modified due to the better carrying capacity to mechanical load, but it is greatly restricted by the cyclic current density. From the view of general performance under electrochemical-mechanical load, particle with 3um diameter presents better mechanical stability under large range of electrochemical-mechanical condition and this particle size is recommended in NCM material preparation.

$$
\frac{P D^{\Delta}}{P_{0} D_{0}{ }^{\Delta}}
$$

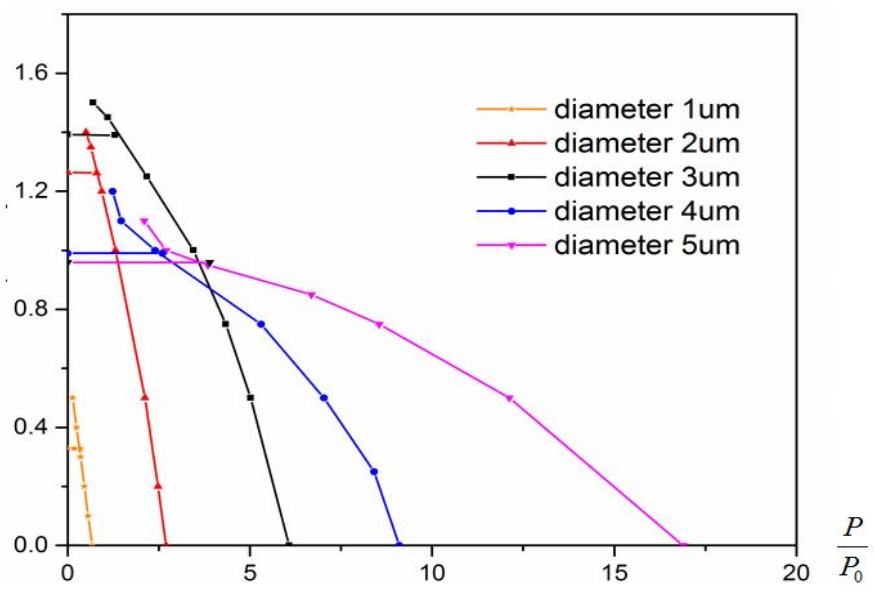

Fig. 11 Ratcheting and shakedown limit curves for electrode particle with various dimensions under coupled cyclic mechanical load - current density and constant mechanical load 


\section{Conclusion}

In the present work, we have comprehensively analyzed the cyclic plasticity behaviours of electrode particle under electrochemical-mechanical condition by employing the shadedown analysis, direct steady cycle analysis and ratchet analysis using the corresponding Linear Matching Methods. The coupled diffusion-stress analyses are performed with the developed ABAQUS subroutines as the basic work for failure assessment. Three categories of structural response, known as shakedown, reverse plasticity and ratcheting, are detailedly studied for primary electrode particle. The critical failure diagrams are established for electrode particle with considering the variation of particle diameter for avoiding the rapid material failure and related performance deterioration.

With the calculation of different combinations of cyclic current density and mechanical load by using the LMM, the shakedown boundaries of electrode particle with various dimensions are established. It is observed as general trends that, the endurance of electrode particle against current density, which leads to the generation of cyclic diffusion-induced stress, will decrease with an increase of particle dimension. On the contrary, larger particle is proved to be able to withstand greater mechanical load. The aforementioned mechanisms codetermine the evolution trends of shakedown boundaries for particles with various dimensions. For verifying the accuracy of the results from the LMM, ABAQUS step-by-step analysis is adopted to observe structural response under certain loading conditions.

For the configuration subjected to loading history out of the shakedown limit, two failure scenarios are investigated, known as low-cycle fatigue failure and ratcheting failure. By using the direct steady cycle analysis in the LMM, it is accessible to efficiently assess the plastic strain range and identify the most dangerous location for particle under different loading conditions at steady state. Associating with the presented low-cycle fatigue model, we evaluate the low-cycle fatigue damage of electrode particle under representative loading cycles. Two different forms of ratcheting boundaries of electrode particle are established to clarify the influence from cyclic current density as well as mechanical load on cyclic plasticity limits, and avoid the rapidly plastic collapse of electrode particle. It is concluded that, the particle with 3um diameter presents relatively better mechanical stability under large range of electrochemical-mechanical load and is recommended in the preparation of NCM cathode material. These results present a comprehensive understanding of cyclic plasticity failure of electrode particle under electrochemical-mechanical condition.

\section{Acknowledgments}

The authors gratefully acknowledge the supports from the China Scholarship Council, Shanghai Automobile Industry Science and Technology Development Foundation (1801), National Natural Science Foundation of China (51828501), the Higher Education Discipline Innovation Project (111 Project) under the funding code B13020, University of Strathclyde and East China University of Science and Technology during the course of this work.

\section{Reference}

[1] V. Etacheri, R. Marom, R. Elazari, G. Salitra, D. Aurbach. Challenges in the development of advanced Li-ion batteries: a review. Energy Environmental Science, 2011, 4, 3243.

[2] M. Armand, J.M. Tarascon. Building better batteries. Nature, 2008, 451(7179):652-657.

[3] S. Amiri, X. Chen, A. Manes, M. Giglio. Investigation of the mechanical behavior of lithium-ion batteries by an indentation technique. International Journal of Mechanical Sciences, 2016, 105:1-10 
[4] A. Mukhopadhyay, B.W. Sheldon. Deformation and stress in electrode materials for Li-ion batteries. Progress in Materials Science, 2014, 63, 58-116.

[5] A. Walk, M. Huttin, M. Kamlah. Comparison of a phase-field model for intercalation induced stresses in electrode particles of lithium ion batteries for small and finite deformation theory. European Journal of Mechanics - A/Solids, 2014, 48: 74-82.

[6] H. Wu, Z. Xie, Y. Wang, C. Lu, Z. Ma. Modeling diffusion-induced stress on two-phase lithiation in lithium-ion batteries. European Journal of Mechanics - A/Solids, 2018, 71: 320-325.

[7] J. Christensen, J. Newman. Stress generation and fracture in lithium insertion materials. Journal of Solid State Electrochemistry, 2006, 10, 293.

[8] X.C. Zhang, W. Shyy, A.M. Sastry. Numerical simulation of intercalation-induced stress in Li-ion battery electrode particles. Journal of The Electrochemical Society, 2007, 154(10), A910-A916.

[9] L. Weng, J. Zhou, R. Cai. Analytical model of Li-ion diffusion-induced stress in nanowire and negative Poisson's ratio electrode under different operations. International Journal of Mechanical Sciences, 2018, 141: 245-261.

[10] Z. Cui, F. Gao, J. Qu. A finite deformation stress-dependent chemical potential and its applications to lithium ion batteries. Journal of the Mechanics and Physics of Solids, 2012, 60(7):1280-1295.

[11] A.M. Korsunsky, T. Sui, B. Song. Explicit formulae for the internal stress in spherical particles of active material within lithium ion battery cathodes during charging and discharging. Materials and Design, 2015, 69: 247-252.

[12] X. Zhu, Y. Chen, H. Chen, W. Luan. The diffusion induced stress and cracking behaviour of primary particle for Li-ion battery electrode. International Journal of Mechanical Sciences, 2020, 178: 105608.

[13] Y. An, H. Jiang. A finite element simulation on transient large deformation and mass diffusion in electrodes for lithium ion batteries. Modelling and Simulation in Materials Science and Engineering, 2013, 21: 074007.

[14] K. Zhang, Y. Li, B. Zheng, G. Wu, J. Wu, F. Yang. Large deformation analysis of diffusion-induced buckling of nanowires in lithium-ion batteries. International Journal of Solids and Structures, 2017, 108: 230-243.

[15] Y. Li, K. Zhang, B. Zheng, F. Yang. Effect of local velocity on diffusion-induced stress in largedeformation electrodes of lithium-ion batteries. Journal of Power Sources, 2016, 319: 168-177.

[16] J. Wen, Y. Wei, Y. Cheng. Examining the validity of Stoney-equation for in-situ stress measurements in thin film electrodes using a large-deformation finite-element procedure. Journal of Power Sources, 2018, 387: 126-134.

[17] X. Zhang, A. Krischok, C. Linder. A variational framework to model diffusion induced large plastic deformation and phase field fracture during initial two-phase lithiation of silicon electrodes. Computer Methods in Applied Mechanics and Engineering, 2016, 312: 51-77.

[18] K.J. Zhao, W.L. Wang, J. Gregoire, M. Pharr, Z.G. Suo, J.J. Vlassak, E. Kaxiras. Lithium-assisted plastic deformation of silicon electrodes in lithium-ion batteries: a first-principles theoretical study. Nano Letters, 2011, 11: 2962-2967.

[19] L. Brassart, K. Zhao, Z. Suo. Cyclic plasticity and shakedown in high-capacity electrodes of lithiumion batteries. International Journal of Solids and Structures, 2013, 50: 1120-1129. 
[20] H. Haftbaradaran, H. Gao. Ratcheting of silicon island electrodes on substrate due to cyclic intercalation. Applied Physics Letters, 2012, 100: 121907.

[21] C. Xu, L. Weng, B. Chen, L. Ji, J. Zhou, R. Cai, S. Lu. Modeling of the ratcheting behavior in flexible electrodes during cyclic deformation. Journal of Power Sources, 2020, 446: 227353.

[22] L. Weng, C. Xu, B. Chen, J. Zhou, R. Cai, Y. Shi. A comparative study on ratcheting deformation between negative Poisson's ratio electrode and thin film electrode in Li-ion battery cyclic operation. Mechanics of Materials, 2020, 150: 103567.

[23] Bree, J., Elastic-plastic behaviour of thin tubes subjected to internal pressure and intermittent high-heat fluxes with application to fast-nuclear-reactor fuel elements.The Journal of Strain Analysis for Engineering Design, 1967. 2(3): p. 226-238

[24] Y.H. Liu, V. Carvelli, G. Maier. Integrity assessment of defective pressurized pipelines by direct simplified methods. International Journal of Pressure Vessels and Piping, 1997, 74: 49-57.

[25] D.K. Vu, A.M. Yan, H. Nguyen-Dang. A primal-dual algorithm for shakedown analysis of structures. Computer Method in Applied Mechanics and Engineering, 2004, 193: 4663-4674.

[26] M. Staat, M. Heitzer. LISA a European project for FEM-based limit and shakedown analysis. Nuclear Engineering and Design, 2001, 206: 151-166.

[27] J. Abou-Hanna, T.E. McGreevy. A simplified ratchetting limit method based on limit analysis using modified yield surface. International Journal of Pressure Vessels and Piping, 2011, 88: 11-18.

[28] R. Seshadri. Inelastic evaluation of mechanical and structural components using the generalized local stress strain method of analysis. Nuclear Engineering and Design, 1995, 153 (2-3): 287-303.

[29] D. Mackenzie, J.T. Boyle, R. Hamilton. The elastic compensation method for limit and shakedown analysis: a review. Journal of Strain Analysis, 2000, 35 (3): 171-188

[30] H.F. Chen, A.R.S. Ponter, R.A. Ainsworth. The linear matching method applied to the high temperature life integrity of structures, Part 1: assessments involving constant residual stress fields. International Journal of Pressure Vessels and Piping, 2006, 83 (2): 123-135

[31] H.F. Chen, A.R.S. Ponter, R.A. Ainsworth. The linear matching method applied to the high temperature life integrity of structures, Part 2: assessments beyond shakedown involving changing residual stress fields. International Journal of Pressure Vessels and Piping, 2006, 83 (2): 136-147

[32] Koiter, W.T. General theorems for elastic plastic solids. Progress in Solid Mechanics, 1960, pp. 167-221.

[33] Melan, E. Theorie statisch unbestimmter Systeme aus ideal-plastichem Baustoff. Sitzungsber. 1936. D. Akad. D. Wiss., Wien 2A (145), 195-218.

[34] X.C. Zhu, H.F. Chen, F.Z. Xuan, X.H. Chen. Cyclic plasticity behaviors of steam turbine rotor subjected to cyclic thermal and mechanical loads. European Journal of Mechanics - A/Solids, 2017, 66: 243-255.

[35] N.K. Cho, H.F. Chen. Shakedown, ratchet, and limit analyses of $90^{\circ}$ back-to-back pipe bends under cyclic in-plane opening bending and steady internal pressure. European Journal of Mechanics A/Solids, 2018, 67: 231-242. 
[36] J. Ure, H.F. Chen, D.J. Tipping. Verification of the linear matching method for limit and shakedown analysis by comparison with experiments. Journal of Pressure Vessel Technology, 2015, 137 (3): 031003-1-031003-6.

[37] D.J. Tipping. The Linear Matching Method: a Guide to the ABAQUS User Subroutines. E/REP/BBGB/0017/GEN/07, British Energy Generation, 2007.

[38] R.A. Ainsworth (Ed.), R5: Assessment Procedure for the High Temperature Response of Structures, vol 3, British Energy Generation Ltd, 2003.

[39] F. Yang. Diffusion-induced stress in inhomogeneous materials: concentration-dependent elastic modulus. SCIENCE CHINA Physics, Mechanics \& Astronomy, 2012, 55, 6: 955-962.

[40] S.L. Zhang. Chemomechanical modeling of lithiation-induced failure in high-volume-change electrode materials for lithium ion batteries. Computational Materials, 2017, 3:7

[41] R. Grantab, V.B. Shenoy. Location- and orientation-dependent progressive crack propagation in cylindrical graphite electrode particles. Journal of The Electrochemical Society, 2011, 158(8):A948

[42] H. Hibbitt, B. Karlsson, P. Sorensen, 2012. ABAQUS Theory Manual, Version 6.12. Pawtucket, Rhode Island, USA.

[43] H. Chen, A. Ponter. A direct method on the evaluation of ratchet limit. Journal of Pressure Vessel Technology, 2010, 132(4): 041202.

[44] R. Xu, H. Sun, L. Vasconcelos, K. Zhao. Mechanical and structural degradation of LiNixMnyCozO2 cathode in Li-ion Batteries: An Experimental study. Journal of Electrochemical Society, 2017, 164(13):A3333-A3341

[45] A. Verma, K. Smith, S. Santhanagopalan, D. Abraham. K. Yao, P. Mukherjee. Galvanostatic intermittent titration and performance based analysis of LiNi0.5Co0.2Mn0.3O2 cathode. Journal of The Electrochemical Society, 2017, 164(13):A3380-A3392

[46] O. Dolotko, A. Senyshyn, M.J. Muhlbauer, K. Nikolowski, H. Ehrenberg. Understanding structural changes in NMC Li-ion cells by in situ neutron diffraction. Journal of Power Sources, 2014, 255:197203

[47] A. Sarkar, P. Shrotriya, A. Chandra. Simulation-driven selection of electrode materials based on mechanical performance for Lithium-Ion battery. Materials, 2019, 12, 831.

[48] R. Xu, L. Vasconcelos, K. Zhao. Computational analysis of chemomechanical behaviors of composite electrodes in Li-ion batteries. Journal of Materials Research, 2016, 31(18), 2715-2727.

[49] J.C.M. Li. Physical chemistry of some microstructural phenomena. Metallurgical Materials Transactions A, 1978, 9:1353

[50] X. Zhu, Y. Xie, H. Chen, W. Luan. Numerical analysis of the cyclic mechanical damage of Li-ion battery electrode and experimental validation. International Journal of Fatigue, 2021, 142: 105915. 